\title{
Time window for postoperative reactive enhancement after resection of brain tumors: less than 72 hours
}

\author{
Stephanie Lescher, M.D., ${ }^{1}$ Sonja Schniewindt, Cand.Med., ${ }^{1}$ \\ Alina Jurcoane, M.D., Ph.D., ${ }^{*}$ Christian Senft, M.D., Ph.D., 2 \\ and Elke Hattingen, M.D. ${ }^{1}$ \\ ${ }^{1}$ Institute of Neuroradiology and ${ }^{2}$ Department of Neurosurgery, Hospital of Goethe University, Frankfurt am \\ Main, Germany
}

Object. Early postoperative MRI within 72 hours after brain tumor surgery is commonly used to assess residual contrast-enhancing tumor. The 72-hour window is commonly accepted because previous 1.5-T MRI studies have not found confounding postoperative reactive contrast enhancement in this time frame. The sensitivity to detect contrast enhancement increases with the field strengths. Therefore, the authors aimed to assess whether the 72-hour window is also appropriate for the MRI scanner with a field strength of $3 \mathrm{~T}$.

Methods. The authors retrospectively analyzed findings on early postsurgical MR images acquired in 46 patients treated for high-grade gliomas. They performed 3-T MRI within 7 days before surgery and within 72 hours thereafter. The appearance of enhancement was categorized as postoperative reactive enhancement or tumoral enhancement by comparison with the pattern and location of presurgical enhancing tumor.

Results. Postoperative reactive enhancement was present in 15 patients $(32.6 \%)$. This enhancement, not seen on presurgical MRI, had a marginal or leptomeningeal/dural pattern. In 13 patients $(28.3 \%)$ postsurgical enhancement was found within the first 72 postoperative hours, with the earliest seen 22:57 hours after surgery. Subsequent MR scans in patients with postoperative reactive enhancement did not reveal tumor recurrence in these regions.

Conclusions. Postoperative reactive enhancement earlier than 72 hours after brain tumor surgery can be expected in about one-third of the cases in which a 3-T scanner is used. This might be due to the higher enhancement-to-brain contrast at higher field strengths. Therefore, the time window of 72 hours does not prevent reactive enhancement, which, however, can be recognized as such comparing it with presurgical enhancing tumor.

(http://thejns.org/doi/abs/10.3171/2014.9.FOCUS14479)

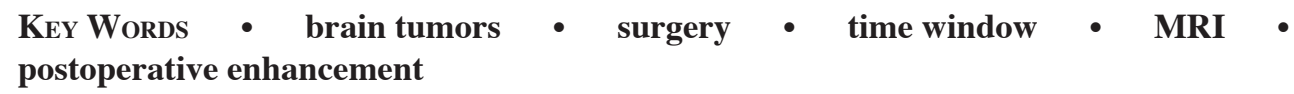

I

$\mathrm{N}$ patients with malignant gliomas, the poor prognosis is determined by the extent of tumor removed, tumor histopathology, age, and symptoms of the patient. ${ }^{2,9,10,30}$ The resection of an enhancing tumor (grosstotal resection) is known to prolong progression-free and overall survival time of patients..$^{32,35,36}$ Most tumor progressions are detected within the 1st year after surgery; thus, accurate postoperative assessment is important. .,21,23 $^{2}$ In the past, the extent of surgical tumor removal was estimated by the neurosurgeons.,10,26 By allowing a comparison with the preoperative MRI findings, postoperative MRI, in recent years, has become the method of choice for determining the presence of any residual tumor. ${ }^{10}$

Abbreviation used in this paper: $\mathrm{iMRI}=$ intraoperative MRI.

* Drs. Senft and Hattingen share senior authorship of this work.
Early postoperative MRI (within 72 hours) is commonly used to determine if a complete tumor resection has been achieved or to show how much enhancing tumor remains in situ. ${ }^{2}$ This postoperative MRI study is the basis for making postsurgical decisions and monitoring the effects of subsequent adjuvant therapy. The time window of 72 hours after surgery is recommended to avoid occurrence of nonneoplastic contrast enhancement due to surgical manipulation.2,18 Early postoperative enhancement and tumor-induced contrast enhancement can look very much alike. ${ }^{12,15}$ Therefore, benign postoperative enhancement can be difficult to distinguish from that caused by residual tumor. Because MRI studies have shown that reactive enhancement does not occur within 72 hours after surgery, enhancement within this time window indicates the presence of residual tumor tissue., ${ }^{2,14}$ The MRI stud- 


\section{S. Lescher et al.}

ies in the past were performed at field strengths of $1.5 \mathrm{~T}$. Today, MRI scanners with higher field strengths (mainly $3 \mathrm{~T}$ ) are becoming more widely available. The magnetic field dependence of contrast enhancement in brain lesions including brain tumors has been addressed in several studies. $1,8,11,17$ The intravenous application of a standard dose of Gd-based contrast medium produces greater contrast between the lesion and normal brain at 3.0 T compared with $1.5 \mathrm{~T}^{27}$ The $\mathrm{T} 1$ relaxation rate of both the nonenhancing brain tissue and contrast-enhancing tissue decreases with increasing field strength, reducing the T1 effect on T1weighted images. However, the overall signal increases at $3 \mathrm{~T}$, and the lower background contrast between gray and white matter yields a higher sensitivity to detect contrastenhancing lesions. ${ }^{4,27,37}$ Until now, there has been no published systematic analysis of the reliability of the 72-hour window as it applies to 3-T MRI. Therefore, we aimed to evaluate if this timeframe is also appropriate for the 3-T MR images. To this end, we retrospectively analyzed the clinical and MRI data of patients with high-grade gliomas who underwent tumor resection.

\section{Methods}

This retrospective noninterventional study was approved by the local institutional review board.

\section{Patient Population}

We retrospectively analyzed the MRI studies of 46 patients with high-grade gliomas who underwent tumor surgery at our institution between January 2012 and June 2013. MRI scans were acquired using a 3-T scanner within 7 days before surgery and within 72 hours after surgery, with identical parameters at both imaging sessions.

Tumor removal was performed with the objective of excising the maximum amount tumor while preserving neurological functions (e.g., motor function, language, and visual function). The surgically resected gliomas were classified histopathologically based on WHO criteria for the classification of CNS tumors. ${ }^{22}$

\section{Imaging Technique}

High-field 3-T MRI (Verio, Siemens) was performed in all patients using a standard protocol, including an axial T2-weighted turbo-spin echo sequence (TR 5.170 msec, TE $92 \mathrm{msec}$ ), axial FLAIR sequence (TR 6.630 msec, TE $=131 \mathrm{msec}$, TI $2.140 \mathrm{msec}$ ), and T2*-weighted images (TR $631 \mathrm{msec}$, TE $20 \mathrm{msec}$ ). Axial T1-weighted spin echo sequences (TR $590 \mathrm{msec}$, TE $12 \mathrm{msec}$, slice thickness $5 \mathrm{~mm}$ ) were acquired before and after intravenous application of a standard dose of Gd-DTPA (0.1 $\mathrm{mmol} / \mathrm{kg}$ ). Furthermore, coronal contrast-enhanced T1weighted gradient echo sequences (TR $261 \mathrm{msec}$, TE 4.12 msec) and contrast-enhanced T1-weighted 3D gradient echo sequence (TR $4.9 \mathrm{msec}$, TE $1.83 \mathrm{msec}$, slice thickness $1 \mathrm{~mm}$ ) were added for analysis. All patients were shown to have had enhancing tumors preoperatively.

\section{Image Analysis}

All imaging studies, including the first serial follow- up scans after the postoperative MR scans (routinely obtained 8 weeks after surgery) were evaluated qualitatively by two experienced neuroradiologists, one with more than 10 years and the other with more than 5 years experience in tumor diagnosis, independently and blinded to the results of the serial follow-up scans.

For qualitative analysis the appearance of enhancement pattern was assessed as it was described by Forsyth et al. ${ }^{14}$ The different patterns of enhancement at the surgical site were categorized as follows: 1) postoperative reactive marginal or dural/leptomeningeal (fine linear areas of enhancement along the operative bed); and 2) tumoral enhancement corresponding to the location of enhancing tumor documented before surgery. Additionally, we compared noncontrast and contrast-enhanced T1-weighted images to identify hyperintense areas on nonenhanced T1-weighted images and to avoid misinterpretation as contrast enhancement. ${ }^{24}$ These hyperintense areas correspond to methemoglobin, whose formation is accelerated by styptic agents.

Postoperative reactive marginal or leptomeningeal/ dural enhancement was defined as fine linear areas of enhancement without any nodularity or bulky areas of enhancement. Similarly, enhancement was defined as residual tumor enhancement if persisting in an area where an enhancing tumor mass was documented before surgery; was bulky, nodular, or mass-like in shape; and was continuously detectable on subsequent follow-up examinations.

The terms used to characterize the scans are descriptive but widely accepted and used by neuroradiologists. We compared clinical and radiological data in all patients, and the follow-up scans reassured us that the fine linear areas of enhancement were not residual tumor, and therefore histological proof was not necessary.

\section{Results}

We examined a total of 46 patients (33 male; mean age 62.5 years, range $26-79$ years) with newly diagnosed malignant gliomas who underwent either gross-total or partial tumor resection.

Histopathological analysis demonstrated glioblastomas in 44 patients, anaplastic astrocytoma (WHO Grade III) in 1 patient, and gliosarcoma in 1 patient.

An intraoperative MRI (iMRI)-guided procedure with "double dose" application of Gd-DTPA (0.2 mmol/ $\mathrm{kg}$ ) was performed in 21 patients. Patients for whom iMRI guidance was used received contrast agent twice per surgery. In 25 patients no iMRI guidance was used.

Postsurgical MR scans were acquired within the first 72 hours after surgery in 41 patients (mean 35:40 hours, range 22:57-56:08 hours) and after 72 hours in 5 patients (mean 86:50 hours, range 73:46-107:33 hours).

On the nonenhanced T1-weighted images, hyperintense areas (methemoglobin) of different degrees and forms were present in the resection site of all patients.

\section{Postoperative Reactive Enhancement}

Postoperative reactive enhancement patterns (marginal or leptomeningeal/dural enhancement) were noted 


\section{Shorter time window after brain tumor surgery using 3-T MRI}

in $15(32.6 \%)$ of 46 patients -7 patients in whom iMRI guidance was used and 8 patients in whom it was. In 6 $(40 \%)$ of these 15 patients additional residual tumor was also present in a portion of the resection cavity distant from the site of the early postoperative reactive enhancement; 1 of these patients had undergone an iMRI-guided procedure.

Reactive enhancement was present within the first 72 hours in $13(28.3 \%)$ of 46 patients (mean 34:30 hours). The earliest occurrence of marginal enhancement was within 22:57 hours after surgery. In 2 patients marginal enhancement occurred beyond 72 hours after surgery (mean 91:27 hours).

Subsequent MR scans in these patients with reactive enhancement on the first postoperative scan after surgery demonstrated no evidence that this enhancement was residual tumor, and no tumor recurrence occurred in these regions.

In our series, 1 of the 3 patients examined within 24 hours following surgery exhibited reactive enhancement not attributable to residual tumor. None of these patients had undergone surgery with iMRI.

Between 24 and 48 hours postoperatively, 6 (35.3\%) of 17 iMRI group and $3(23.1 \%)$ of 13 in the non-iMRI group exhibited reactive enhancement.

Beyond 48 hours postoperatively, 1 (25.0\%) of 4 patients in the iMRI group and $5(55.6 \%)$ of 9 patients in the non-iMRI group had evidence of reactive enhancement.

\section{iMRI-Guided Surgery}

Seven of the 13 patients with postoperative reactive enhancement within the 72-hour window had undergone iMRI-guided tumor resection in which Gd was applied intraoperatively. The percentage of patients with postoperative reactive enhancement did not differ between the patients with and without iMRI (33\% and 32\%, respectively). In the 7 patients who underwent iMRI-guided surgery and who had postoperative reactive enhancement, the MRI had been performed within a mean of $34: 12$ hours after surgery (range 25:35-53:34 hours). In the 8 patients who did not undergo iMRI-guided surgery, the postsurgical MRI was done within a mean of 49:35 hours (range 22:57-107:33 hours).

\section{Residual Tumor Enhancement}

Enhancement of residual tumor in areas also evident on the presurgical scans was present in 31 patients $(67.4 \%)$. None of these patients underwent a second surgery or stereotactic biopsied. Nine of the 31 patients had received a contrast agent intraoperatively for iMRI.

\section{Discussion}

We were able to show that reactive contrast enhancement after resection of high-grade gliomas can appear within the 72-hour window on MR images obtained using at 3-T scanner in a not insignificant number of patients. Our results differ from those of previous studies in which images were acquired at lower field strength and revealing no contrast enhancement within the first
72 hours after surgery. ${ }^{2,13,14,32}$ MRI showed postoperative enhancement patterns in 15 of our 46 patients, in 13 of whom evidence was found within the 72-hour window (mean time window within 34:30 hours). Therefore, field strength may be the one important factor influencing the visual detection of reactive enhancement. In contrast, the application of an intraoperative contrast agent does not influence the occurrence of benign enhancement postoperatively. In both iMRI and non-iMRI groups, postoperative reactive enhancement appeared within 72 hours after surgery in one-third of the patients. However, it should be noted that this enhancement occurred earlier if the patient received an intraoperative contrast agent before the postsurgical MR scan (mean 34:12 hours with Gd-based iMRI vs 49:35 hours without Gd-based iMRI), raising the question of whether preceding application of contrast agent additionally narrows the time window for the occurrence of postoperative reactive enhancement. To answer this question, prospective studies will be required. The use of iMRI has increased in recent years, because it has been shown to increase the percentage of the complete tumor resection compared with surgeries without iMRI. 7,29,32-34

Furthermore, it would be very helpful to fix an optimal time frame for the postoperative MRI. Our data suggest that reactive enhancement after surgery varies greatly in terms of time and appearance, although the surgical procedure was standardized irrespective of the use of iMRI. The marked heterogeneity of glioblastoma, their anatomical environment, and the presence of residual tumor influence surgical procedure (e.g., the use of hemostatic material or cautery), which in turn may result in a different manner of enhancement. In addition, the manufacturers of MRI scanners, dose and type of contrast agent administered, and the sequence parameters also influence the visibility of contrast enhancement. Therefore, even if we could provide optimal time frame for conducting postoperative MRI at our institution, there is no guarantee that our time frame would be suitable for other institutions because of these variables.

The authors of previous MRI studies have investigated postsurgical changes, aiming to identify the optimal time after surgery to assess benign and malignant contrast enhancement findings. . $^{12-14,16,18,28,31}$ However, the detection of enhancement strongly depends on various factors, such as imaging sequence parameters, magnetic field strength, contrast agent dose, and manner of contrast agent administration. ${ }^{6,20}$ Since the 1994 publication by Albert et al., the opinion has been that benign enhancement occasionally appears on Day 4 after surgery but never before that. ${ }^{2}$ This benign enhancement has been explained by postsurgical repair mechanisms at the resection site that result from hypervascularization and disruption of blood-brain barrier. Postoperative benign linear enhancement can be observed for up to 3 months after treatment, and meningeal enhancement often persists even longer. ${ }^{2}$ As a result of the study by Albert et al., the standard has been to perform postsurgical MRI in patients within the first 72 hours after glioma resection. Thus, misinterpretation of residual tumor and benign postoperative reactive enhancement should be avoided.

Although our data did not reach statistical signifi- 
cance, they suggest that reactive enhancement may appear earlier when contrast-enhanced iMRI precedes the postoperative MRI. Neurosurgeons and radiologists should pay special attention to this association in the future.

In line with our results, Ekinci et al. reported 25 of 30 cases in which 1.5-T MRI documented enhancement in the resection bed within the first 24 hours without evidence of residual tumor tissue. ${ }^{10}$ The authors distinguished the postoperative enhancement patterns, with thick nodular enhancement corresponding to residual tumor and thin linear enhancement corresponding to benign contrast enhancement. Therefore, one should question the generally accepted belief that the "diagnostic window" for performing MRI is within 72 hours after surgery.

The reason for this discrepancy can only be assumed. The higher field strengths used in our study might not be the only reason for the shortened time window for detection of postoperative reactive enhancement, since Ekinci et al. did use a 1.5-T scanner. Additionally, other technical advantages such as new head coils with better signal-to-noise performance and also the parameters of the T1-weighted sequence might influence detectability of the benign enhancement. $3,19,25,38,39$

However, confounding findings from early reactive enhancement may be limited if the imaging criteria of this enhancing pattern are considered. In our study, the types of enhancement shown on the first postoperative
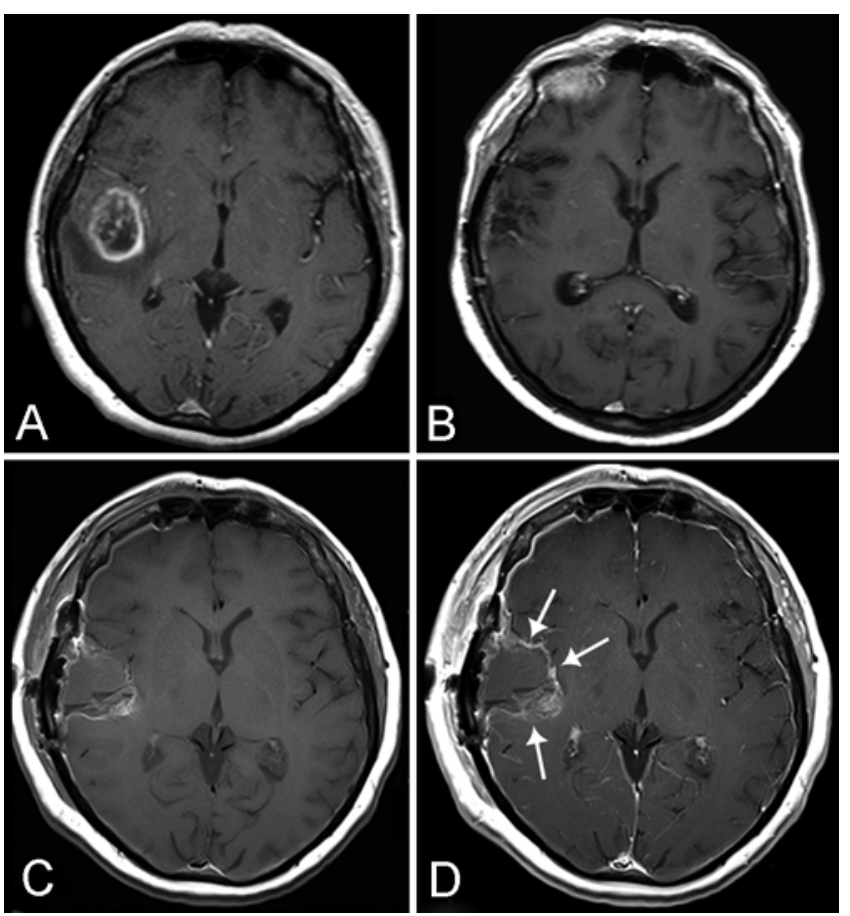

FIG. 1. The first postoperative MRI scans were acquired 53:30 hours after surgery in a patient with a glioblastoma in the right temporal lobe. Preoperative T1-weighted images with (A) and without (B) contrast agent. Surgery was performed with the aim of achieving total tumor resection. Noncontrast (C) and contrast-enhanced (D) T1-weighted images demonstrating fine linear areas of enhancement at the margins of the resection cavity (arrows) on the postcontrast image. Nodular or bulky enhancement suggesting residual tumor was not present. The subsequent MR scan in this patient did not reveal any tumor recurrence in these regions on contrast-enhanced T1-weighted images (D). reactive MR scan could easily be attributed to reactive enhancement or residual gross tumor by comparing preand postsurgical scans, the latter revealing identical parts of the presurgical enhancing tumor. In cases of enhancement exhibiting a nodular or mass-like shape, the type of enhancement itself was highly suggestive of residual gross tumor (Fig. 1). The real type of this enhancement was confirmed by follow-up examinations. Examples of early postoperative reactive enhancement included linear enhancement along the resection margins as well as leptomeningeal or dural enhancement (Fig. 2). Early dural and leptomeningeal enhancement usually appeared near the craniotomy site or at intergyral and interlobar interfaces. In some patients, new gyriform enhancement of the adjacent parenchyma occurred after surgery. This was the result of ischemia, and the lesion turned into parenchymal defects on the follow-up MRI examinations.

Limitations of this study are its retrospective design and the use of a single field strength only. However, even when comparing results from different field strengths, one should consider other confounding factors such as the coil characteristics, sequence parameters, type of
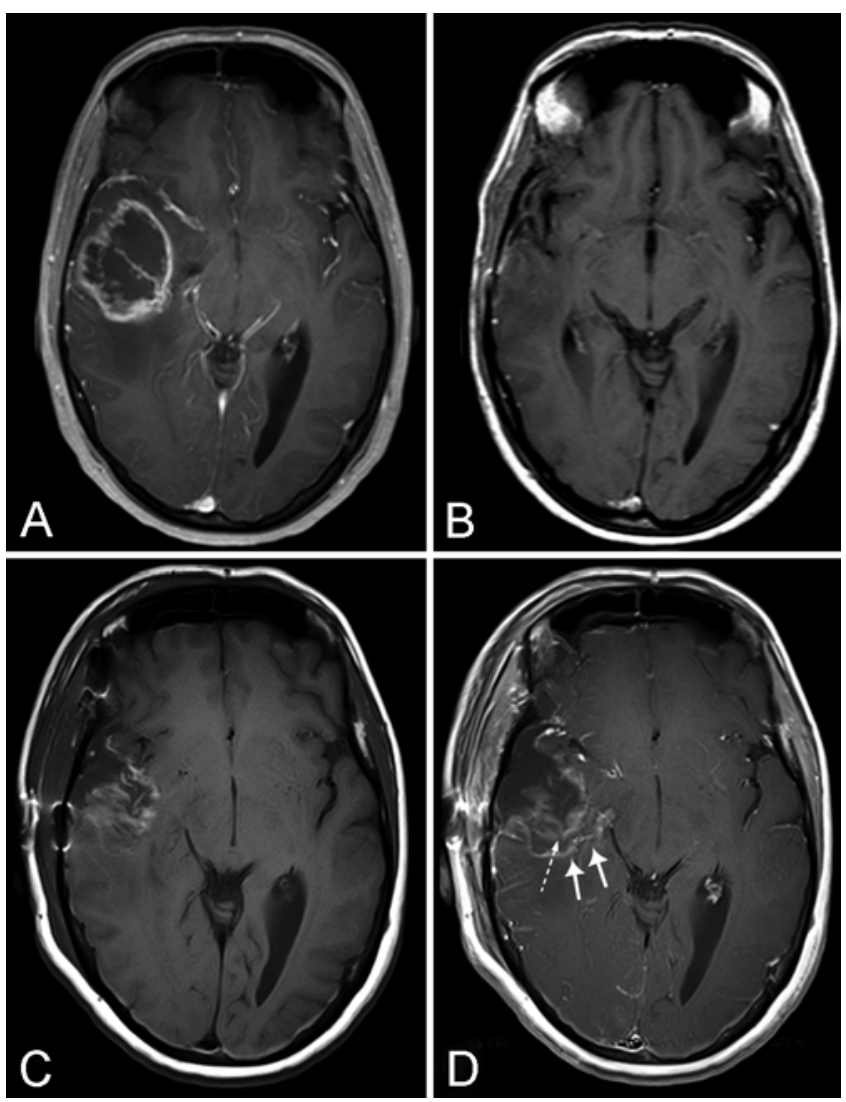

FIG. 2. The first postoperative MRI scans were obtained 28:00 hours after surgery in a different patient with a glioblastoma in the right temporal lobe. Preoperative T1-weighted images with (A) and without (B) contrast agent. Surgery was performed with the aim of achieving total tumor resection. Comparison of the preoperative images with noncontrast (C) and contrast-enhanced (D) postoperative T1-weighted images identified fine linear areas of enhancement at the mediodorsal margins of the resection cavity on the postcontrast T1-weighted image (D; arrows). Moreover, some hemorrhage was seen that was around the resection cavity (dotted arrow). 
contrast agent, and differences among the MRI scanners from various manufactures. Additionally, the study lacks statistical and systematical analyses to establish an exact definition of the optimal time window and to generalize conclusions.

Furthermore, we used descriptive parameters to define the types of enhancement. This approach was still supported by the inspection of follow-up MR images showing no recurrent tumor in the areas of marginal enhancement.

\section{Conclusions}

Benign postoperative reactive enhancement after glioma resection can be found earlier than the generally accepted period of 72 hours after surgery. We observed postoperative nonneoplastic enhancement in 13 (28.3\%) of 46 patients within the first 72 postoperative hours; the earliest enhancement was noted 22:57 hours after surgery. The higher field strengths $(3 \mathrm{~T})$ might be one reason for the shorter interval, but the factors influencing the detectability of contrast enhancement are manifold and difficult to standardize. Nonetheless, the critical visual analysis of enhancing patterns, together with the comparison of postsurgical with presurgical MR images, seems to enable discrimination between residual tumor and benign reactive enhancements.

\section{Disclosure}

Dr. Senft reports receiving honoraria as an invited speaker from Medtronic Navigation. The other authors report no conflict of interest.

Author contributions to the study and manuscript preparation include the following. Conception and design: Lescher, Hattingen. Acquisition of data: Lescher, Schniewindt. Analysis and interpretation of data: Lescher. Drafting the article: Lescher. Critically revising the article: Jurcoane, Senft, Hattingen. Study supervision: Hattingen.

\section{References}

1. Akeson P, Vikhoff B, Ståhlberg F, Holtås S: Brain lesion contrast in MR imaging. Dependence on field strength and concentration of gadodiamide injection in patients and phantoms. Acta Radiol 38:14-18, 1997

2. Albert FK, Forsting M, Sartor K, Adams HP, Kunze S: Early postoperative magnetic resonance imaging after resection of malignant glioma: objective evaluation of residual tumor and its influence on regrowth and prognosis. Neurosurgery 34:4561,1994

3. Anzalone N, Essig M, Lee SK, Dörfler A, Ganslandt O, Combs $\mathrm{SE}$, et al: Optimizing contrast-enhanced magnetic resonance imaging characterization of brain metastases: relevance to stereotactic radiosurgery. Neurosurgery 72:691-701, 2013

4. Ba-Ssalamah A, Nöbauer-Huhmann IM, Pinker K, Schibany N, Prokesch R, Mehrain S, et al: Effect of contrast dose and field strength in the magnetic resonance detection of brain metastases. Invest Radiol 38:415-422, 2003

5. Bonnin JM, Garcia JH: Histology and growth characteristics of brain neoplasms, in Taveras JM, Ferrucci JT (eds): Radiology: Diagnosis, Imaging, Intervention. Philadelphia: JB Lippincott, 1991, pp 1-11

6. Brück W, Bitsch A, Kolenda H, Brück Y, Stiefel M, Lassmann $\mathrm{H}$ : Inflammatory central nervous system demyelination: correlation of magnetic resonance imaging findings with lesion pathology. Ann Neurol 42:783-793, 1997
7. Burtscher IM, Skagerberg G, Geijer B, Englund E, Ståhlberg F, Holtås S: Proton MR spectroscopy and preoperative diagnostic accuracy: an evaluation of intracranial mass lesions characterized by stereotactic biopsy findings. AJNR Am J Neuroradiol 21:84-93, 2000

8. Chang KH, Ra DG, Han MH, Cha SH, Kim HD, Han MC: Contrast enhancement of brain tumors at different MR field strengths: comparison of $0.5 \mathrm{~T}$ and $2.0 \mathrm{~T}$. AJNR Am J Neuroradiol 15:1413-1423, 1994

9. Cohadon F: Indications for surgery in the management of gliomas. Adv Tech Stand Neurosurg 17:189-234, 1990

10. Ekinci G, Akpinar IN, Baltacioğlu F, Erzen C, Kiliç T, Elmaci $\mathrm{I}$, et al: Early-postoperative magnetic resonance imaging in glial tumors: prediction of tumor regrowth and recurrence. Eur J Radiol 45:99-107, 2003

11. Elster AD: Field-strength dependence of gadolinium enhancement: theory and implications. AJNR Am J Neuroradiol 15: 1420-1423, 1994

12. Elster AD, DiPersio DA: Cranial postoperative site: assessment with contrast-enhanced MR imaging. Radiology 174:93-98, 1990

13. Forsting M, Albert FK, Kunze S, Adams HP, Zenner D, Sartor K: Extirpation of glioblastomas: MR and CT follow-up of residual tumor and regrowth patterns. AJNR Am J Neuroradiol 14:77-87, 1993

14. Forsyth PA, Petrov E, Mahallati H, Cairncross JG, Brasher P, MacRae ME, et al: Prospective study of postoperative magnetic resonance imaging in patients with malignant gliomas. J Clin Oncol 15:2076-2081, 1997

15. Glantz MJ, Hoffman JM, Coleman RE, Friedman AH, Hanson MW, Burger PC, et al: Identification of early recurrence of primary central nervous system tumors by $[18 \mathrm{~F}]$ fluorodeoxyglucose positron emission tomography. Ann Neurol 29: 347-355, 1991

16. Haba D, Pasco Papon A, Tanguy JY, Burtin P, Aube C, CaronPoitreau C: Use of half-dose gadolinium-enhanced MRI and magnetization transfer saturation in brain tumors. Eur Radiol 11:117-122, 2001

17. Haustein J, Laniado M, Niendorf HP, Hilbertz T, Planitzer J, Schörner W, et al: Administration of gadopentetate dimeglumine in MR imaging of intracranial tumors: dosage and field strength. AJNR Am J Neuroradiol 13:1199-1206, 1992

18. Henegar MM, Moran CJ, Silbergeld DL: Early postoperative magnetic resonance imaging following nonneoplastic cortical resection. J Neurosurg 84:174-179, 1996

19. Kakeda S, Korogi Y, Hiai Y, Ohnari N, Moriya J, Kamada K, et al: Detection of brain metastasis at 3T: comparison among SE, IR-FSE and 3D-GRE sequences. Eur Radiol 17:23452351, 2007

20. Katz D, Taubenberger JK, Cannella B, McFarlin DE, Raine CS, McFarland HF: Correlation between magnetic resonance imaging findings and lesion development in chronic, active multiple sclerosis. Ann Neurol 34:661-669, 1993

21. Kelly WM, Brant-Zawadzki B: Magnetic resonance imaging and computed tomography of supratentorial tumors, in Taveras JM, Ferrucci JT (eds): Radiology: Diagnosis, Imaging, Intervention. Philadelphia: JB Lippincott, 1991, pp 1-22

22. Louis DN, Ohgaki H, Wiestler OD, Cavenee WK, Burger PC, Jouvet A, et al: The 2007 WHO classification of tumours of the central nervous system. Acta Neuropathol 114:97-109, 2007

23. Madison MT, Hall WA, Latchaw RE, Loes DJ: Radiologic diagnosis, staging, and follow-up of adult central nervous system primary malignant glioma. Radiol Clin North Am 32: 183-196, 1994

24. Meyding-Lamadé U, Forsting M, Albert F, Kunze S, Sartor $\mathrm{K}$ : Accelerated methaemoglobin formation: potential pitfall in early postoperative MRI. Neuroradiology 35:178-180, 1993

25. Nagai A, Shibamoto Y, Mori Y, Hashizume C, Hagiwara M, 


\section{S. Lescher et al.}

Kobayashi T: Increases in the number of brain metastases detected at frame-fixed, thin-slice MRI for gamma knife surgery planning. Neuro Oncol 12:1187-1192, 2010

26. Nazzaro JM, Neuwelt EA: The role of surgery in the management of supratentorial intermediate and high-grade astrocytomas in adults. J Neurosurg 73:331-344, 1990

27. Nöbauer-Huhmann IM, Ba-Ssalamah A, Mlynarik V, Barth M, Schöggl A, Heimberger K, et al: Magnetic resonance imaging contrast enhancement of brain tumors at 3 tesla versus 1.5 tesla. Invest Radiol 37:114-119, 2002

28. Oser AB, Moran CJ, Kaufman BA, Park TS: Intracranial tumor in children: MR imaging findings within 24 hours of craniotomy. Radiology 205:807-812, 1997

29. Rabin BM, Meyer JR, Berlin JW, Marymount MH, Palka PS, Russell EJ: Radiation-induced changes in the central nervous system and head and neck. Radiographics 16:1055-1072, 1996

30. Salcman M: Survival in glioblastoma: historical perspective. Neurosurgery 7:435-439, 1980

31. Sato N, Bronen RA, Sze G, Kawamura Y, Coughlin W, Putman $\mathrm{CM}$, et al: Postoperative changes in the brain: MR imaging findings in patients without neoplasms. Radiology 204:839846, 1997

32. Schucht P, Murek M, Jilch A, Seidel K, Hewer E, Wiest R, et al: Early re-do surgery for glioblastoma is a feasible and safe strategy to achieve complete resection of enhancing tumor. PLoS One 8:e79846, 2013

33. Schwartz RB, Hsu L, Wong TZ, Kacher DF, Zamani AA, Black PM, et al: Intraoperative MR imaging guidance for intracranial neurosurgery: experience with the first 200 cases. Radiology 211:477-488, 1999

34. Senft C, Bink A, Franz K, Vatter H, Gasser T, Seifert V: Intraoperative MRI guidance and extent of resection in glioma surgery: a randomised, controlled trial. Lancet Oncol 12:9971003, 2011

35. Stummer W, Pichlmeier U, Meinel T, Wiestler OD, Zanella F, Reulen HJ: Fluorescence-guided surgery with 5-aminolevulinic acid for resection of malignant glioma: a randomised controlled multicentre phase III trial. Lancet Oncol 7:392-401, 2006

36. Stummer W, Reulen HJ, Meinel T, Pichlmeier U, Schumacher W, Tonn JC, et al: Extent of resection and survival in glioblastoma multiforme: identification of and adjustment for bias. Neurosurgery 62:564-576, 2008

37. Trattnig S, Pinker K, Ba-Ssalamah A, Nöbauer-Huhmann IM: The optimal use of contrast agents at high field MRI. Eur Radiol 16:1280-1287, 2006

38. Welker KM, Tsuruda JS, Hadley JR, Hayes CE: Radio-frequency coil selection for MR imaging of the brain and skull base. Radiology 221:11-25, 2001

39. Yuh WT, Tali ET, Nguyen HD, Simonson TM, Mayr NA, Fisher DJ: The effect of contrast dose, imaging time, and lesion size in the MR detection of intracerebral metastasis. AJNR Am J Neuroradiol 16:373-380, 1995

Manuscript submitted August 13, 2014.

Accepted September 8, 2014.

Please include this information when citing this paper: DOI: 10.3171/2014.9.FOCUS14479.

Address correspondence to: Stephanie Lescher, M.D., Institute of Neuroradiology, Hospital of Goethe University, Schleusenweg 2-16, 60528 Frankfurt am Main, Germany. email: stephanie.lescher@ kgu.de. 